\title{
Influence of hormones on the
}

immunotolerogenic molecule HLA-G:

a cross-sectional study in patients with congenital adrenal hyperplasia

\author{
Lee S Nguyen ${ }^{1,2}$, Nathalie Rouas-Freiss ${ }^{3}$, Christian Funck-Brentano ${ }^{1}$, Monique Leban $^{4}$, Edgardo D Carosella ${ }^{3}$, \\ Philippe Touraine $^{4,5}$, Shaida Varnous ${ }^{2}$, Anne Bachelot ${ }^{4}$ and Joe-Elie Salem ${ }^{1,6}$
}

'Department of Pharmacology, Sorbonne Université, INSERM CIC Paris-Est, UNICO AP-HP.6 Cardio-Oncology Program, Pitié-Salpêtrière Hospital, Paris, France, '2Department of Cardiothoracic Surgery, Sorbonne Université, Institute of Cardiology, Pitie-Salpetriere University Hospital, ICAN, Paris, France, ${ }^{3}$ Department of Research in Hemato-Immunology (SRHI), CEA, Saint-Louis Institute, UMR U976, Paris, France, ${ }^{4}$ Department of Endocrinology, Pitie-Salpetriere University Hospital, Sorbonne Universite, ICAN, Paris, France, ${ }^{5}$ Center for Rare Endocrine Disorders and center for Rare Gynecological Disorders, Paris, France, and ${ }^{6}$ Department of Pharmacology and Medicine, Cardio-Oncology Program, Vanderbilt University Medical Center,

Correspondence should be addressed to L S Nguyen Email

nguyen.lee@icloud.com

\begin{abstract}
Background: HLA-G is an immune checkpoint molecule, naturally expressed during pregnancy, playing a critical role in the tolerance of the fetal semi-allograft from the maternal immune system. While HLA-G expression levels are associated with progesterone, the influence of other hormones is still unclear. Congenital adrenal hyperplasia (CAH) represents an adequate model to study the hormonal influence on biomarkers as it leads to impaired cortisol biosynthesis and increased progesterone and androgens production due to 21-hydroxylase enzyme deficiency. Methods: In a cross-sectional study of CAH patients matched on sex and age with healthy control, the association between circulating levels of soluble HLA-G and hormones was assessed by use of non-parametric analyses tests. Multivariable linear regressions were performed on normalized data.

Results: Overall, $83 \mathrm{CAH}$ patients and 69 healthy controls were included. Among CAH patients, all were under glucocorticoid and 52 (62.6\%) were under mineralocorticoid supplementation. Compared to controls, CAH patients had increased HLA-G levels ( $15 \mathrm{vs} 8 \mathrm{ng} / \mathrm{mL}, P=0.02$ ). In controls, HLA-G level was independently associated with progesterone and estradiol $(\beta=0.44(0.35-1.27)$ and $-0.44(-0.94,-0.26)$ respectively, both $P$ values $=0.001)$. In CAH patients, HLA-G level was independently associated with mineralocorticoid supplementation dosage $(\beta=0.25$ (0.04-0.41), $P=0.001)$ and estradiol $(\beta=-0.22(-0.57,-0.02), P<0.001)$.

Conclusion: CAH patients had higher HLA-G levels than healthy controls. HLA-G level was positively associated with progesterone and corticosteroid supplementation, and negatively with estradiol. The association between mineralocorticoid, renin and HLA-G levels may suggest a role of the renin-angiotensin system in the expression of soluble HLA-G.
\end{abstract}

European Journal of

(2019) 181, 481-488 


\section{Introduction}

Human leucocyte antigens (HLA) are cell-surface proteins coded by the major histocompatibility complex (MHC). HLA-G is a type Ib non-classical molecule featuring (i) a more restricted polymorphism than other HLA, with only 19 protein variants, (ii) the existence of seven protein isoforms (soluble or transmembrane) generated from alternative splicing of the primary HLA-G transcript, and (iii) the ability to induce immunotolerance instead of activating non-self-recognition cascades. The latter feature is a characteristic of pregnancy, a situation of tolerance toward the fetal semi-allograft. Hence HLA-G is closely associated with immunomodulatory properties and is considered as an immune checkpoint molecule $(1,2,3)$. Studies in obstetrics were the first to show the existence of HLA-G and a strong association between HLA-G and progesterone, with an increased risk of miscarriage and preeclampsia in patients with lower HLA-G expression $(4,5,6)$. However, the influence of other hormones on HLA-G is unclear. HLA-G can be found at the cell surface or circulating in body fluids as membrane-bound or soluble proteins, respectively. Whether membrane bound or in soluble isoform, HLA-G displayed the same immunomodulatory capabilities (7).

Congenital adrenal hyperplasia (CAH) represents an adequate model to study the hormonal influence over biomarkers. Indeed, CAH leads to impaired cortisol biosynthesis due to $21 \alpha$-hydroxylase deficiency leading to increased progesterone and androgens production. Moreover, treatment of $\mathrm{CAH}$ patients relies on glucocorticoid and in some cases mineralocorticoid supplementation.

We hypothesized that hormones and corticosteroid supplementation had an influence on circulating levels of HLA-G. Thus, in a prospective observational cohort study of CAH patients matched on sex and age with healthy controls, we aimed to separately analyze the relationships between steroid hormones and soluble HLA-G levels.

\section{Patients and methods}

\section{Study design}

This study is ancillary to the Cardiovascular Risk Profile in Patients With Congenital Adrenal Hyperplasia (CARDIOHCS) study (clinicaltrials.gov identifier NCT01807364), a multicenter prospective observational study comparing early cardiovascular damage in adult men and women with $\mathrm{CAH}$ due to $21 \alpha$-hydroxylase deficiency and healthy control subjects. All patients provided written informed consent to participate, and the study was approved by each hospital ethics committee.

\section{Study population and setting}

Inclusion criteria were patients with $\mathrm{CAH}$, aged more than 18 years, referred by one of three university hospital endocrinology departments (Pitie-Salpetriere University Hospital and Saint-Antoine University Hospital (Paris, France); and Kremlin-Bicetre University Hospital (Le Kremlin-Bicetre, France)).

For every patient with $\mathrm{CAH}$, a healthy control matched for sex, age, and smoking status was prospectively included in the CARDIOHCS study (NCT01807364) between May 2011 and August 2015 (8, 9, 10). Patients with $\mathrm{CAH}$ and healthy controls were all assessed at the Clinical Investigation Center Paris-Est (CIC-1421, PitieSalpetriere University Hospital, Paris, France). All CAH patients had been identified prior to the study, during childhood and confirmed by genetic testing of CYP21A2 gene. Healthy controls were recruited via specialized ads. All underwent full medical check-up (medical history, physical examination, standard blood sampling related to the study).

$\mathrm{CAH}$ patients were categorized in one of three clinical presentation types: classic salt-wasting, simple virilizing or non-classical form (11).

Exclusion criteria for $\mathrm{CAH}$ and healthy subjects were known history of cardiovascular disease and pregnancy. Estradiol and/or progesterone contraception in the previous month was an exclusion criterion in healthy subjects, and temporary discontinuation was promoted in patients with $\mathrm{CAH}$.

\section{Exposure to corticosteroid supplementation in the $\mathrm{CAH}$ group}

All patients with CAH were treated, as needed by their standard of care, with hydrocortisone or dexamethasone. Some of them received fludrocortisone in association.

Dosage of hydrocortisone equivalent regarding the glucocorticoid effect (hereafter named total glucocorticoid supplementation) was computed as the sum of dosage of hydrocortisone equivalent and 0.0154 times the dosage of fludrocortisone.

Dosage of fludrocortisone equivalent regarding the mineralocorticoid effect (hereafter named total mineralocorticoid supplementation) was computed as 
the sum of dosage of fludrocortisone and five times the dosage of hydrocortisone equivalent (12).

\section{Hormone analyses}

All blood samples were collected at one fixed timepoint in the morning and patients were required to be fasting for at least $12 \mathrm{~h}$ and to refrain from any coffee-containing product for at least $24 \mathrm{~h}$ prior to blood sampling.

Blood samples for the determination of serum concentrations of, progesterone, $17-\mathrm{OH}$ progesterone, estradiol, total testosterone, renin, delta 4 androstenedione, sex hormone-binding globulin (SHBG), adrenocorticotropic hormone (ACTH), folliclestimulating hormone (FSH), luteinizing hormone (LH), were collected in a dry tube and further assayed in the immunology laboratory of Pitie-Salpetriere University Hospital. Estradiol, progesterone, SHBG, testosterone, ACTH, FSH, LH were assayed using chemiluminescence (Modular-E170; Roche()), renin by chemiluminescence (Liaison XL; Diasorin ()) and 17-OH progesterone, delta 4 androstenedione by radioimmunoassay (KIP1409; DIAsource $@$ ImmunoAssays, Louvain-la-Neuve, Belgium and RIA delta4 -Androstenedione; Beckman-Coulter ( respectively).

\section{HLA-G analysis}

HLA-G was analyzed in plasma of patients as follows: soluble HLA-G was quantified using MEM-G/9, which recognizes the most abundant soluble isoforms (sHLA-G1, -G5) and anti-human $\beta 2$-microglobulin as capture and detection antibodies, respectively (13). Total sHLA-G levels were determined from a five-point standard curve (12.5-200 ng/mL) using dilutions of calibrated HLA-G5 purified from M8-HLA-G5 cell line culture supernatant, and the results were expressed as $\mathrm{ng} / \mathrm{mL}$. The limit of detection is $1 \mathrm{ng} / \mathrm{mL}$. The methodology to measure HLA-G using ELISA has been validated previously (14).

\section{Statistical analysis}

Using Shapiro-Wilk and D'Agostino and Pearson omnibus normality tests, the only variables which were normally distributed were height and blood pressure. Hence, continuous variables are presented as median (interquartile range) and categorical variables as number (proportions). Comparisons were performed using nonparametric tests due to data distribution. Consequently, associations were tested using Spearman correlations.
In order to perform multivariable linear regressions, data were normalized using logarithmic transformation. Multivariable linear regression was performed using a stepwise model with entry and removal criteria a probability of $\mathrm{F}<0.05$ and $>0.10$, respectively. Assuming a correlation between progesterone and HLA-G levels of 0.35 , a minimum of 62 patients would be required to achieve statistical significance, given a type I error rate of 0.05 and type II error rate of 0.2 . All calculations, statistical analyses and figures were executed using SPSS 25.0 (IBM).

\section{Results}

\section{Overall population}

One hundred fifty-two subjects were included in this study, 83 of whom had CAH (54.6\%). Patients with CAH and healthy controls were adequately matched and similar in age and sex. Clinical and biological characteristics including hormone levels are presented in Table 1 . There were no missing data.

Among the CAH patients, 41 (49.4\%) presented the classic salt-wasting form, 16 (19.3\%) the simple virilizing form and 26 (31.3\%) were non-classical. Among CAH patients, $50(60.2 \%)$ may be considered controlled with normal androstenedione levels (below $4 \mathrm{ng} / \mathrm{mL}$ ). Among CAH men $(n=25), 9$ (36\%) had testicular adrenal rest tumors. Median dosage of glucocorticoid supplementation was 21 (interquartile range: 15-32) $\mathrm{mg}$ daily and in the 52 patients $(62.6 \%)$ under mineralocorticoid supplementation, median dosage was 200 (75-245) $\mu \mathrm{g}$ daily. In comparison, none of the healthy controls was under corticoid treatment. Fifteen patients (18.1\%) were receiving other steroids: six women were on cyproterone acetate treatment for anti-androgenic property, two on progestin only pills (desogestrel), two on sequential progestin (dydrogesterone and one chlormadinone), one on combined oral contraceptive with desobel and two men under mitotane (for testicular adrenal rest tumors). Two (2.4\%) CAH women were under menopause as compared to $3(4.3 \%)$ in healthy controls.

$\mathrm{CAH}$ patients had higher body-mass index (BMI) than healthy controls (25.6 vs 23.2, $P=0.02)$. Compared to healthy controls, CAH patients had increased levels of progesterone and 17-OH progesterone, testosterone (total, bioavailable and free), ACTH and renin (Table 1).

Circulating HLA-G levels were higher in patients with CAH (median value: 15 (interquartile range: $4-25) \mathrm{ng} / \mathrm{mL}$ ) than in healthy controls $(8(3-22) \mathrm{ng} / \mathrm{mL})(P=0.02)$. 
Table 1 Clinical and biological characteristics of $\mathrm{CAH}$ and healthy control patients. Data are presented as median and interquartile range (first quartile: Q1, third quartile: Q3) for continuous variables and number (proportion) for categorical variables.

\begin{tabular}{|c|c|c|c|}
\hline & \multicolumn{3}{|c|}{ Healthy controls $(n=69)$} \\
\hline & Median & Q1 & Q3 \\
\hline Women & 49 & $(71 \%)$ & \\
\hline Age (years) & 27.8 & 23.4 & 35 \\
\hline Height (cm) & 170 & 163 & 178 \\
\hline Weight (kg) & 69 & 61 & 77 \\
\hline BMI & 23.23 & 20.7 & 25.85 \\
\hline SBP (mmHg) & 111 & 103 & 118 \\
\hline $\mathrm{DBP}(\mathrm{mmHg})$ & 69 & 63 & 72 \\
\hline CRP $(\mathrm{u} / \mathrm{L})$ & 0.71 & 0.4 & 1.4 \\
\hline Glycemia (mmol/L) & 4.5 & 4.3 & 4.7 \\
\hline Total cholesterol (mmol/L) & 4.45 & 3.91 & 4.95 \\
\hline HDL cholesterol (mmol/L) & 1.55 & 1.24 & 1.89 \\
\hline LDL cholesterol (mmol/L) & 2.45 & 2.1 & 2.8 \\
\hline Triglycerides (mmol/L) & 0.72 & 0.55 & 0.92 \\
\hline Estradiol (pg/mL) & 59 & 28.4 & 140 \\
\hline Progesterone (ng/mL) & 0.8 & 0.5 & 1.2 \\
\hline $\mathrm{FSH}(\mathrm{IU} / \mathrm{mL})$ & 4.8 & 3.5 & 7.6 \\
\hline LH (IU/mL) & 5.2 & 4.3 & 8.8 \\
\hline Androstenedione (ng/mL) & 2.2 & 1.7 & 3 \\
\hline 17-OH progesterone $(\mathrm{ng} / \mathrm{mL})$ & 1.39 & 0.86 & 2.2 \\
\hline Total testosterone (ng/mL) & 0.36 & 0.26 & 4.5 \\
\hline SHBG $(n g / m L)$ & 58.1 & 36.3 & 79 \\
\hline Free androgen index & 2.32 & 1.01 & 44.23 \\
\hline ACTH $(p g / m L)$ & 19 & 11.1 & 29 \\
\hline $\operatorname{Renin}(\mathrm{pg} / \mathrm{mL})$ & 11.8 & 8.8 & 15.8 \\
\hline Cortisol ( $\mu \mathrm{g} / \mathrm{L})$ & 97.3 & 65.2 & 138 \\
\hline HLA-G (ng/mL) & 8 & 3 & 21 \\
\hline
\end{tabular}

\begin{tabular}{|c|c|c|c|}
\hline \multicolumn{3}{|c|}{ CAH patients $(n=83)$} & \multirow[b]{2}{*}{$P$ value } \\
\hline Median & Q1 & Q3 & \\
\hline 58 & (69.9\%) & & 0.88 \\
\hline 27.4 & 22.6 & 36 & 1 \\
\hline 162 & 156 & 168 & $<0.001$ \\
\hline 67 & 57 & 74 & 1 \\
\hline 25.64 & 21.97 & 28.6 & 0.02 \\
\hline 110 & 103 & 123 & 0.95 \\
\hline 68 & 59 & 75 & 0.88 \\
\hline 0.53 & 0.3 & 1.39 & 0.46 \\
\hline 4.4 & 4.2 & 4.7 & 0.21 \\
\hline 4.56 & 4.04 & 5.46 & 0.5 \\
\hline 1.48 & 1.14 & 1.79 & 0.53 \\
\hline 2.76 & 2.29 & 3.35 & 0.02 \\
\hline 0.87 & 0.62 & 1.13 & 0.02 \\
\hline 47 & 31 & 117 & 0.27 \\
\hline 2 & 0.7 & 6.4 & $<0.001$ \\
\hline 4.8 & 3 & 6.5 & 0.88 \\
\hline 5.2 & 2.7 & 7.7 & 0.91 \\
\hline 2.8 & 1.1 & 6.3 & 0.33 \\
\hline 14.3 & 4.3 & 49 & $<0.001$ \\
\hline 0.81 & 0.26 & 2.9 & 0.001 \\
\hline 48.7 & 30.1 & 81.9 & 0.19 \\
\hline 6.04 & 1.59 & 30.23 & 0.03 \\
\hline 31.1 & 10.4 & 89 & 0.003 \\
\hline 21.7 & 13.8 & 42.7 & $<0.001$ \\
\hline 108 & 63.7 & 143 & 0.16 \\
\hline 15 & 4 & 25 & 0.02 \\
\hline
\end{tabular}

17-OH progesterone, 17-hydroxyprogesterone; ACTH, adrenocorticotropic hormone; BMI, body mass index; CAH, congenital adrenal hyperplasia; CRP, C-reactive protein; DBP, diastolic blood pressure; FSH, follicle-stimulating hormone; HDL, high-density lipoprotein; HLA, human leukocyte antigen; LDL, low-density lipoprotein; LH, luteinizing hormone; SBP, systolic blood pressure; SHBG, sex hormone-binding globulin.

\section{Relationship between treatment and hormones in CAH patients}

Glucocorticoid supplementation dosage was positively correlated with renin (correlation coefficient rho $=0.29$, $P=0.009$ ) and negatively correlated with androstenedione (rho $=-0.34, \quad P=0.001$ ). $\quad$ Mineralocorticoid supplementation dosage was positively correlated with renin (rho $=0.27, P=0.016)$ and negatively correlated with progesterone $(\mathrm{rho}=-0.23, P=0.038)$ and androstenedione (rho $=-0.35, P=0.001)$.

There were no correlations between treatment and cortisol, ACTH, total testosterone, estradiol, FSH, LH and 17-OH progesterone (see Supplementary Table 1, see section on supplementary data given at the end of this article).

\section{Relationship between hormones and HLA-G}

In healthy controls, HLA-G levels correlated with progesterone $(\mathrm{rho}=0.25, P=0.04)$ and estradiol levels (rho=-0.25, $P=0.04 ;$ Table 2). Multivariable linear regression (accounting for age, gender, progesterone, estradiol, androstenedione, testosterone, cortisol, ACTH and renin) showed that HLA-G levels were associated with progesterone and estradiol levels (adjusted beta coefficient $\beta=0.44$ (95\% confidence interval for $\beta$ : $0.35-1.27)$ and $\beta=-0.44(-0.94,-0.26)$, respectively; both $P$ values $=0.001)$ (see Fig. 1).

In CAH patients, HLA-G levels correlated with glucocorticoid supplementation (rho=0.27, $P=0.01$ ), mineralocorticoid supplementation (rho $=0.25, P=0.03$ ), renin (rho $=0.23, P=0.036)$, estradiol $(\mathrm{rho}=-0.3, P=0.01)$ and androstenedione levels (rho $=-0.23, P=0.04$ ) (see Table 3). Multivariable linear regression (accounting for age, gender, estradiol, androstenedione, testosterone, cortisol, ACTH, renin, mineralocorticoids and glucocorticoids) showed that HLA-G levels were independently associated with mineralocorticoid supplementation $(\beta=0.25$ (0.04-0.41), $\quad P=0.001)$ and with estradiol $(\beta=-0.22$ $(-0.57,-0.02), \quad P<0.001)$ (see Fig. 1). As additional 
Table 2 Association between HLA-G, hormones and corticosteroid supplementation in healthy controls ( $n=68)$ (non-parametric correlations).

\begin{tabular}{|c|c|c|c|c|c|c|}
\hline & \multicolumn{2}{|c|}{ Healthy controls all $(n=69)$} & \multicolumn{2}{|c|}{ Healthy men $(n=20)$} & \multicolumn{2}{|c|}{ Healthy women $(n=49)$} \\
\hline & Correlation coefficient & $P$ value & Correlation coefficient & $P$ value & Correlation coefficient & $P$ value \\
\hline Estradiol & -0.25 & 0.04 & -0.26 & 0.27 & -0.25 & 0.09 \\
\hline Progesterone & 0.25 & 0.04 & -0.08 & 0.74 & 0.34 & 0.02 \\
\hline $\mathrm{FSH}$ & -0.16 & 0.2 & -0.2 & 0.41 & -0.11 & 0.45 \\
\hline LH & -0.12 & 0.33 & -0.21 & 0.37 & -0.07 & 0.66 \\
\hline Androstenedione & 0.06 & 0.65 & -0.19 & 0.43 & 0.11 & 0.46 \\
\hline 17-OH progesterone & 0.15 & 0.21 & -0.22 & 0.35 & 0.24 & 0.1 \\
\hline Total testosterone & 0.13 & 0.29 & -0.25 & 0.29 & 0.19 & 0.18 \\
\hline SHBG & -0.07 & 0.59 & -0.06 & 0.79 & -0.027 & 0.85 \\
\hline FAI & 0.1 & 0.4 & -0.29 & 0.21 & 0.15 & 0.31 \\
\hline АCTH & 0.04 & 0.76 & -0.24 & 0.32 & 0.13 & 0.39 \\
\hline Renin & 0.04 & 0.76 & 0.08 & 0.75 & 0.02 & 0.89 \\
\hline
\end{tabular}

17-OH progesterone, 17-hydroxyprogesterone; ACTH, adrenocorticotropic hormone; FSH, follicle-stimulating hormone; LH, luteinizing hormone; SHBG, sex hormone-binding globulin.

sensitivity analysis, in CAH patients, after exclusion of patients under anticontraceptive or antiandrogen drug, associations remained unchanged in unadjusted analysis: renin (rho $=0.26, P=0.03$ ), estradiol (rho $=-0.26$, $P=0.03), \quad$ androstenedione $\quad($ rho $=-0.30, \quad P=0.01)$,
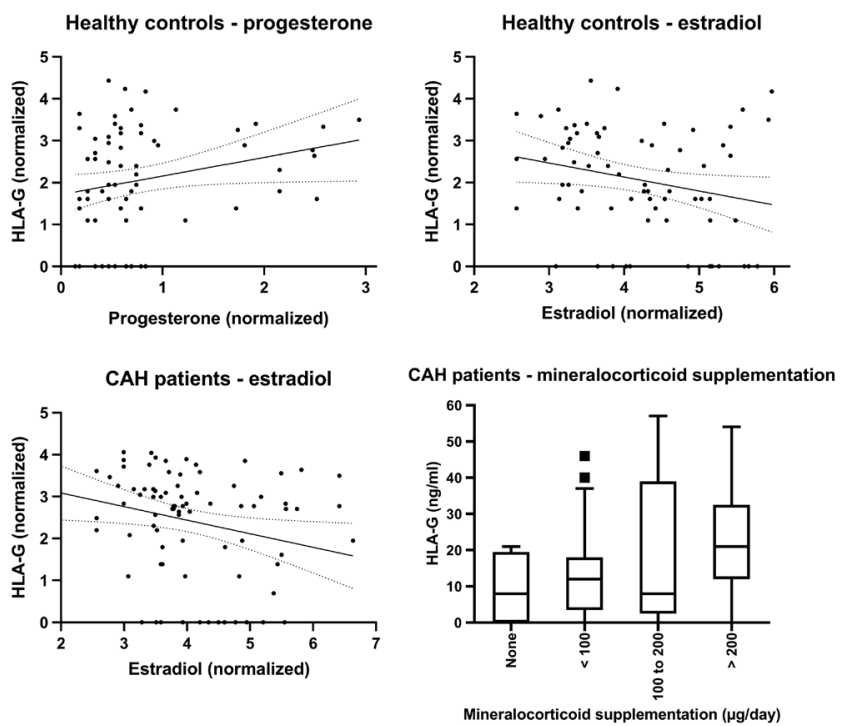

\section{Figure 1}

Association of soluble HLA-G with hormones and steroids levels. Top left: scatterplot of progesterone and HLA-G levels in healthy controls $(n=68)$ (adjusted $\beta=0.44(0.35-1.27)$, $P=0.001)$; top right: scatterplot of estradiol and HLA-G levels in healthy controls $(n=68)$ (adjusted $\beta=-0.44(-0.94,-0.26)$, $P=0.001)$; bottom left: scatterplot of estradiol and HLA-G levels in $\mathrm{CAH}$ patients $(n=83)$ (adjusted $\beta=-0.22(-0.57$, $-0.02), P<0.001$ ) and bottom right: Tukey plot of mineralocorticoid supplementation and HLA-G levels in CAH patients $(n=83)$ (adjusted $\beta=0.25(0.04-0.41), P=0.001)$. glucocorticoid supplementation (rho $=0.35, P=0.003)$ and mineralocorticoid supplementation (rho $=0.34, P=0.004$ ). After adjustment, the only remaining independent association was with mineralocorticoid supplementation ( $\beta=0.34(0.11-0.53), P=0.004)$.

\section{Discussion}

The main findings of this study are as follows: (i) $\mathrm{CAH}$ patients express more circulating HLA-G than matched healthy controls; (ii) in healthy controls, HLA-G levels were independently associated with progesterone and estradiol levels; (iii) HLA-G levels were associated to renin levels and (iv) in CAH patients, HLA-G levels were correlated with glucocorticoid supplementation and mineralocorticoid supplementation, but only the latter was independently associated with HLA-G levels.

To our knowledge, this is the first time that HLA-G was examined in $\mathrm{CAH}$ patients, and the finding that they express more circulating HLA-G than matched healthy controls is surprising. It has to be noted that while the median value of HLA-G levels in CAH patients was twice as high as that of healthy controls, interquartile range was close between the two groups; hence, patients presented similar outer bound values in both groups. Difference between the two groups was however sufficient to be statistically significant in non-parametric comparison $(P=0.02)$.

The link between progesterone and HLA-G levels was described previously in obstetrics $(5,6,15)$. In other fields, and notably in cardiac transplantation, progesterone could induce expression of HLA-G by cardiomyocytes and coronary endothelial cells, with a reversible 
Table 3 Association between HLA-G, hormones and corticosteroid supplementation in CAH patients $(n=83)($ non-parametric correlations).

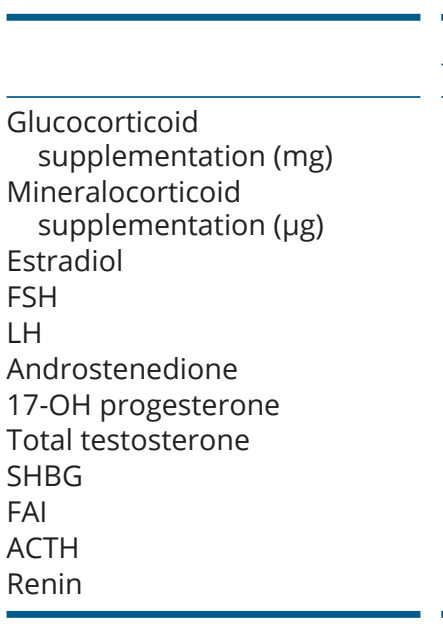

\begin{tabular}{|c|c|}
\hline \multicolumn{2}{|c|}{ CAH all $(n=83)$} \\
\hline Correlation coefficient & $P$ value \\
\hline 0.27 & 0.01 \\
\hline 0.25 & 0.03 \\
\hline-0.30 & 0.01 \\
\hline 0.10 & 0.39 \\
\hline-0.05 & 0.65 \\
\hline-0.23 & 0.04 \\
\hline-0.08 & 0.50 \\
\hline 0.13 & 0.23 \\
\hline-0.06 & 0.56 \\
\hline 0.14 & 0.19 \\
\hline-0.03 & 0.80 \\
\hline 0.23 & 0.04 \\
\hline
\end{tabular}

\begin{tabular}{|c|c|}
\hline \multicolumn{2}{|c|}{ CAH men $(n=25)$} \\
\hline Correlation coefficient & $P$ value \\
\hline 0.11 & 0.59 \\
\hline 0.08 & 0.71 \\
\hline-0.31 & 0.14 \\
\hline 0.15 & 0.46 \\
\hline 0.03 & 0.87 \\
\hline-0.32 & 0.12 \\
\hline-0.05 & 0.83 \\
\hline-0.65 & 0.76 \\
\hline 0.21 & 0.31 \\
\hline-0.34 & 1 \\
\hline-0.003 & 0.51 \\
\hline 0.27 & 0.19 \\
\hline
\end{tabular}

\begin{tabular}{|c|c|}
\hline \multicolumn{2}{|c|}{ CAH women $(n=68)$} \\
\hline Correlation coefficient & $P$ value \\
\hline 0.16 & 0.22 \\
\hline 0.1 & 0.48 \\
\hline
\end{tabular}

17-OH progesterone, 17-hydroxyprogesterone; ACTH, adrenocorticotropic hormone; FSH, follicle-stimulating hormone; LH, luteinizing hormone; SHBG, sex hormone-binding globulin.

process observed when adding progesterone antagonist mifepristone (16). In JEG-3 choriocarcinoma cell line and first-trimester cytotrophoblasts, expression of HLA-G via progesterone stimulation goes through a promoter, the progesterone-responsive element, bound by an activated receptor (6). Response is dose dependent with maximal enhancement of both steady-state mRNA and protein expression. Incidentally, this promoter activity was observed throughout heart wall and vessels (vascular endothelial and smooth muscle cells) which may help understand how HLA-G may be increased even in spite of lack of cytotrophoblasts (i.e. in men).

The lack of association between HLA-G and 17-OH-progesterone levels in our cohort may be explained by the counter-regulation effect of substitutive treatment (with a non-linear effect of corticosteroid effect on levels of 17-OH-progesterone, as attested by the non-significant correlations which were observed), or the fact it has a very mild progestative effect (below 1\%). The relationship between estradiol and HLA-G is less clear than with progesterone. During early phases of pregnancy, both hormones are elevated. Experimental studies failed to demonstrate a beneficial effect of estradiol toward the expression of HLA-G in adipose-derived stem cells, and may even be interpreted as an antagonist effect of estradiol compared with progesterone, which would tend to be confirmed by the present work (17).

In CAH patients, soluble HLA-G levels were positively associated with substitutive treatment, mainly glucocorticoids and mineralocorticoids. Unsurprisingly, both glucocorticoid and mineralocorticoid supplementation were associated with plasma renin. While the effect of glucocorticoids on HLA-G levels has been demonstrated previously (18), the specific effect of mineralocorticoids has not.

Because glucocorticoids (i.e. hydrocortisone) have a five-fold higher mineralocorticoid effect than pure mineralocorticoids (i.e. fludrocortisone), promotion of HLA-G expression may also be influenced by the renin-angiotensin system (RAS), through means of immunomodulatory properties associated to this system $(19,20)$. Incidentally, renin was associated with HLA-G in the whole cohort.

On the one hand, HLA-G expression is influenced by hypoxia, which naturally occurs during the trophoblastic implantation phase in the early placenta development or during invasion of metastatic tumor cells in hypoxic areas $(21,22)$. This hypoxia-related modulation of HLA-G expression relies on the metabolic sensor hypoxia-inducible factor 1 alpha (HIF-1) signaling $(23,24)$. On the other hand, previous experimental studies linked renin-producing cells to hypoxia-responsive elements (namely HIF-2) (25, 26). Moreover, diseases in which RAS is activated (i.e. heart failure with reduced ejection fraction and hypertension) present with increased soluble HLA-G $(27,28)$.

Hence, RAS may be associated with HLA-G through HIF, although the relationship between mineralocorticoids, renin-angiotensin system, HIF and HLA-G would need to be investigated further in a specific experimental setting.

Clinical consequences of the increase of circulating HLA-G observed in CAH patients in this cohort do not explain two aspects of immunomodulation in $\mathrm{CAH}$ 
patients. During pregnancy, CAH women do not present with different outcomes as compared to general population, particularly with regard to preeclampsia events; they do however, have an increased rate of gestational diabetes, imputable to corticoid supplementation (29). No data are available on the risk of rejection after organ transplantation in CAH patients. The lack of clinical proof that HLA-G overexpression may have an incidence in $\mathrm{CAH}$ patients does not refute the findings of the present study, but on the contrary, legitimates future investigations. Given chronic exposure to corticosteroid treatment is associated with increased risk of mortality, HLA-G may be key to identifying patients at higher risk.

\section{Limitations}

Analysis of progesterone in $\mathrm{CAH}$ patients could not be analyzed without bias given that healthy control women were excluded if under contraceptive pills, which was not the case of CAH patients, in whom, a substantial proportion were under contraceptive pills or antiandrogen drugs with progestative effect. Therefore, studying the relationship between endogenous progesterone level and HLA-G in CAH patients would have been subjected to the bias of being under the influence of progestative effect, not assayed to be correctly interpreted.

Other study limitations include the fact that samples were only available at one timepoint, which excludes any dynamic analysis which may have strengthened the results. Moreover, associations between HLA-G and hormones were moderate, although this may be explained by the important number of confounding factors which may alter hormone levels in CAH patients. Biases include the overlapping effect of glucocorticoids into mineralocorticoid effect. To adjust for these, only the associations which resisted multivariate analyses were discussed earlier. Another limitation lies in the method of hormone assay (i.e. steroids), for which, a tandem mass spectrometer analysis was not routinely performed in the course of the CARDIO-HCS study but may have contributed to more precise results regarding the effects of substitutive treatment on HLA-G. Finally, the exploratory nature of these finding, due to the purely observational nature of the study groups is acknowledged.

\section{Conclusion}

CAH patients had higher HLA-G levels than healthy controls. Association with progesterone was confirmed in healthy controls. Other factors associated with HLA-G levels included estradiol, renin and corticosteroid supplementation (glucocorticoid and mineralocorticoid). Renin-angiotensin system involvement in HLA-G expression may be suggested.

\section{Supplementary data}

This is linked to the online version of the paper at https://doi.org/10.1530/ EJE-19-0379.

\section{Declaration of interest}

The authors declare that there is no conflict of interest that could be perceived as prejudicing the impartiality of this study.

\section{Funding}

This research did not receive any specific grant from any funding agency in the public, commercial or not-for-profit sector.

\section{Author contribution statement}

L S Nguyen designed the ancillary study, performed statistical analyses and wrote the manuscript. N Rouas-Freiss, C Funck-Brentano, M Leban, E D Carosella, P Touraine and S Varnous provided critical insight to the manuscript. A Bachelot and J-E Salem supervised the study and provided critical revision to the manuscript.

\section{Acknowledgments}

The authors thank the CARDIOHCS study group, including J Dulon, N Dahmoune, E Bruckert, A Gallo, M Polak, J Leger, S Salenave and $\mathrm{N}$ Bourcigaut. They are also extremely grateful to the study participants.

\section{References}

1 Ferreira LMR, Meissner TB, Tilburgs T \& Strominger JL. HLA-G: at the interface of maternal-fetal tolerance. Trends in Immunology $2017 \mathbf{3 8}$ 272-286. (https://doi.org/10.1016/j.it.2017.01.009)

2 Rebmann V, da Silva Nardi F, Wagner B \& Horn PA. HLA-G as a tolerogenic molecule in transplantation and pregnancy. Journal of Immunology Research $2014 \mathbf{2 0 1 4} 297073$. (https://doi. org/10.1155/2014/297073)

3 Rouas-Freiss N, Goncalves RM, Menier C, Dausset J \& Carosella ED. Direct evidence to support the role of HLA-G in protecting the fetus from maternal uterine natural killer cytolysis. PNAS 199794 11520-11525. (https://doi.org/10.1073/pnas.94.21.11520)

4 Szekeres-Bartho J, Varga P, Kinsky R \& Chaouat G. Progesteronemediated immunosuppression and the maintenance of pregnancy. Research in Immunology 1990141 175-181. (https://doi. org/10.1016/0923-2494(90)90139-P)

5 Szekeres-Bartho J, Polgar B, Kozma N, Miko E, Par G, Szereday L, Barakonyi A, Palkovics T, Papp O \& Varga P. Progesterone-dependent immunomodulation. Chemical Immunology and Allergy 200589 118-125. (https://doi.org/10.1159/000087953)

6 Yie SM, Li LH, Li GM, Xiao R \& Librach CL. Progesterone enhances HLA-G gene expression in JEG-3 choriocarcinoma cells and human cytotrophoblasts in vitro. Human Reproduction 200621 46-51. (https://doi.org/10.1093/humrep/dei305) 
7 Carosella ED, Rouas-Freiss N, Tronik-Le Roux D, Moreau P, LeMaoult J. HLA-G: an immune checkpoint molecule. Advances in Immunology 2015127 33-144. (https://doi.org/10.1016/ bs.ai.2015.04.001)

8 Rosenbaum D, Gallo A, Lethielleux G, Bruckert E, Levy BI, Tanguy ML, Dulon J, Dahmoune N, Salem JE, Bittar R et al. Early central blood pressure elevation in adult patients with 21-hydroxylase deficiency. Journal of Hypertension 201937 175-181. (https://doi.org/10.1097/HJH.0000000000001850)

9 Abehsira G, Bachelot A, Badilini F, Koehl L, Lebot M, Favet C, Touraine P, Funck-Brentano C \& Salem JE. Complex influence of gonadotropins and sex steroid hormones on QT interval duration. Journal of Clinical Endocrinology and Metabolism 2016101 2776-2784. (https://doi.org/10.1210/jc.2016-1877)

10 Salem JE, Nguyen LS, Hammoudi N, Preud'homme G, Hulot JS, Leban M, Funck-Brentano C, Touraine P, Isnard R, Bachelot A et al. Complex association of sex hormones on left ventricular systolic function: insight into sexual dimorphism. Journal of the American Society of Echocardiography 201831 231-240.e1. (https://doi. org/10.1016/j.echo.2017.10.017)

11 El-Maouche D, Arlt W \& Merke DP. Congenital adrenal hyperplasia. Lancet 2017390 2194-2210. (https://doi.org/10.1016/S01406736(17)31431-9)

12 Goodman L, Gilman A, Brunton L, Lazo J \& Parker K. Goodman \& Gilman's the pharmacological basis of therapeutics. 2006 New York: McGraw-Hill. Brunton L. The Pharmalogical Basis of Therapeutics, 2006.

13 Menier C, Saez B, Horejsi V, Martinozzi S, Krawice-Radanne I, Bruel S, Le Danff C, Reboul M, Hilgert I, Rabreau M et al. Characterization of monoclonal antibodies recognizing HLA-G or HLA-E: new tools to analyze the expression of nonclassical HLA class I molecules. Human Immunology 200364 315-326. (https://doi.org/10.1016/s01988859(02)00821-2)

14 Rebmann V, Lemaoult J, Rouas-Freiss N, Carosella ED \& GrosseWilde $\mathrm{H}$. Report of the wet workshop for quantification of soluble HLA-G in Essen, 2004. Human Immunology 200566 853-863. (https://doi.org/10.1016/j.humimm.2005.05.003)

15 Ragusa A, de Carolis C, dal Lago A, Miriello D, Ruggiero G, Brucato A, Pisoni MP, Muscara M, Merati R, Maccario L et al. Progesterone supplement in pregnancy: an immunologic therapy? Lupus 200413 639-642. (https://doi.org/10.1191/0961203304lu20 07oa)

16 Sheshgiri R, Rao V, Tumiati LC, Xiao R, Prodger JL, Badiwala M, Librach C \& Delgado DH. Progesterone induces human leukocyte antigen-g expression in vascular endothelial and smooth muscle cells. Circulation 2008118 S58-S64. (https://doi.org/10.1161/ CIRCULATIONAHA.107.757781)

17 Moslehi A, Hashemi-Beni B, Moslehi A, Akbari MA \& Adib M. The effect of progesterone and 17-beta estradiol on membrane-bound HLA-G in adipose derived stem cells. Korean Journal of Physiology and Pharmacology 201620 341-346. (https://doi.org/10.4196/ kjpp.2016.20.4.341)
18 Moreau P, Faure O, Lefebvre S, Ibrahim EC, O'Brien M, Gourand L, Dausset J, Carosella ED \& Paul P. Glucocorticoid hormones upregulate levels of HLA-G transcripts in trophoblasts. Transplantation Proceedings 200133 2277-2280. (https://doi. org/10.1016/s0041-1345(01)01990-x)

19 Capettini LS, Montecucco F, Mach F, Stergiopulos N, Santos RA $\&$ da Silva RF. Role of renin-angiotensin system in inflammation, immunity and aging. Current Pharmaceutical Design 201218 963-970. (https://doi.org/10.2174/138161212799436593)

20 Satou R, Penrose H \& Navar LG. Inflammation as a regulator of the renin-angiotensin system and blood pressure. Current Hypertension Reports 201820 100-100. (https://doi.org/10.1007/s11906-018-0900-0)

21 Rodesch F, Simon P, Donner C \& Jauniaux E. Oxygen measurements in endometrial and trophoblastic tissues during early pregnancy. Obstetrics and Gynecology 199280 283-285.

22 Kilburn BA, Wang J, Duniec-Dmuchowski ZM, Leach RE, Romero R \& Armant DR. Extracellular matrix composition and hypoxia regulate the expression of HLA-G and integrins in a human trophoblast cell line. Biology of Reproduction 200062 739-747. (https://doi. org/10.1095/biolreprod62.3.739)

23 Mouillot G, Marcou C, Zidi I, Guillard C, Sangrouber D, Carosella ED $\&$ Moreau P. Hypoxia modulates HLA-G gene expression in tumor cells. Human Immunology 200768 277-285. (https://doi. org/10.1016/j.humimm.2006.10.016)

24 Garziera M, Scarabel L \& Toffoli G. Hypoxic modulation of HLA-G expression through the metabolic sensor HIF-1 in human cancer cells. Journal of Immunology Research 20172017 4587520. (https:// doi.org/10.1155/2017/4587520)

25 Kurt B, Gerl K, Karger C, Schwarzensteiner I \& Kurtz A. Chronic hypoxia-inducible transcription factor-2 activation stably transforms juxtaglomerular renin cells into fibroblast-like cells in vivo. Journal of the American Society of Nephrology 201526 587-596. (https://doi. org/10.1681/ASN.2013111152)

26 Stefanska A, Eng D, Kaverina N, Pippin JW, Gross KW, Duffield JS \& Shankland SJ. Cells of renin lineage express hypoxia inducible factor 2alpha following experimental ureteral obstruction. BMC Nephrology 201617 5. (https://doi.org/10.1186/s12882-015-0216-0)

27 Olesen LL \& Hviid TV. Upregulation of soluble HLA-G in chronic left ventricular systolic dysfunction. Journal of Immunology Research 2016 2016 8417190. (https://doi.org/10.1155/2016/8417190)

28 Garcia-Gonzalez IJ, Valle Y, Rivas F, Figuera-Villanueva LE, MunozValle JF, Flores-Salinas HE, Gutierrez-Amavizca BE, DavalosRodriguez NO \& Padilla-Gutierrez JR. The 14 bp Del/Ins HLA-G polymorphism is related with high blood pressure in acute coronary syndrome and type 2 diabetes mellitus. BioMed Research International 20142014 898159. (https://doi.org/10.1155/2014/898159)

29 Hagenfeldt K, Janson PO, Holmdahl G, Falhammar H, Filipsson H, Frisen L, Thoren M \& Nordenskjold A. Fertility and pregnancy outcome in women with congenital adrenal hyperplasia due to 21-hydroxylase deficiency. Human Reproduction 200823 1607-1613. (https://doi.org/10.1093/humrep/den118)

Received 20 May 2019

Revised version received 5 September 2019

Accepted 9 September 2019 